\title{
GUANACOS TÍMIDOS, HUEMULES CONFIADOS: EL LÍMITE OCCIDENTAL DE LOS CAZADORES TERRESTRES AUSTRALES
}

\author{
NORMA I. DÍAZ", ALFREDO PRIETO","*** Y GABRIEL BAHAMONDE*"
}

\begin{abstract}
RESUMEN
Se estudia el estatus de los huemules como presa de los cazadores terrestres del sur de la Patagonia, a partir de los registros históricos de su conducta en la zona precordillerana. Se entregan algunos antecedentes arqueológicos acerca de su consumo, y se analiza si su conducta era el producto de las presiones de caza ejercidas por los grupos humanos de la Patagonia austral.
\end{abstract}

PALABRAS CLAVES: etología, huemules, límites étnicos, cazadores-recolectores.

\section{SHY GUANACOS, TRUSTING HUEMULS: THE WESTERN BOUNDARY OF TERRESTRIAL SOUTHERN HUNTERS-GATHERERS}

\begin{abstract}
Based on historical records for the pre-Andean zone, the paper examines the role of the huemul (Hippocamelus bisulcus) as prey of choice for Southern Patagonian terrestrial hunter-gatherers. We review archaeological evidence for the consumption of these cervids and also explore whether their behavioural patterns can be considered the result of hunting pressure by human communities of Southern Patagonia.
\end{abstract}

KEY WORDS: ethology, cervids, ethnic boundaries, hunter-gatherers.

Según los indios el huemul era "un animal que se había caído de la luna y afirmaban que era tan escaso porque las hembras no tenían sino un hijo en toda su vida, y eso en un año en que hubieran dos eclipses, uno de sol y otro de luna”. (En el Mar Austral, Fray Mocho).

* I.U.C.N. Tupac - Amarú 1011, C1407CXC, Buenos Aires, Argentina.

* Centro de Estudios del Hombre Austral. Instituto de la Patagonia, Universidad de Magallanes. Av. Bulnes 01890, Punta Arenas. Chile. E-mail: alfredo.prieto@umag.cl; gabriel.bahamonde@umag.cl

*entro de Estudios del Cuaternario de Fuego-Patagonia y Antártica (CEQUA). Punta Arenas, Chile. 
El huemul (Hippocamelus bisulcus) no parece haber sido nunca parte importante de la dieta de los cazadores recolectores terrestres de la Patagonia austral, pese a su amplia distribución a lo largo de la cordillera. Sin embargo, no fue así el caso de su utilización por parte de los nómades del mar quienes lo aprovechaban más regularmente (Fig. 1). En la zona continental de la región hay leves indicios de su uso en los niveles medios y tardíos de algunos sitios arqueológicos. No así en la Isla Grande de Tierra del Fuego donde parece no haber habitado, de hecho una de las pocas islas en que habita es la isla Riesco, entre los senos Otway y Skyring, además de las islas Wellington y Stuven.

Lo que nos parece importante y discutiremos aquí, es la actitud de este animal hacia el hombre en algunos sectores precordilleranos de la Patagonia austral. Aquello que se ha dado en llamar naïveté, que traduciremos por ingenuidad, concepto que ha sido utilizado como factor concomitante en la extinción de las especies de algunas regiones del mundo, particularmente de Australasia y América, y en menor medida de África.
La conducta del huemul hacia el hombre podría ser utilizada, desde esta perspectiva, para testear la capacidad de modificación infligida por el hombre en un paisaje naï. Ello requiere adentrarse en el conocimiento de esta especie de una peculiar mansedumbre respecto a otros cérvidos para poder dirimir si la ingenuidad exhibida en los casos que mencionaremos más adelante es producto intrínseco de la conducta intraespecífica o muestra simplemente un área que no era de uso frecuente por parte de los cazadores terrestres. Esto es, una suerte de etnolímite del territorio, o más aún, el límite precordillerano del huemul no fuera más que aquél inducido por el hombre a lo largo de toda la ocupación humana del territorio.

John Hatcher es uno de los primeros que se ocupa de la etología de las especies patagónicas australes en un notable capítulo de su libro. Allí establece la diferencia existente entre la actitud arisca del guanaco (Lama guanicoe) y la extrema confianza del huemul: A la mañana temprano y al atardecer era común ver a los ciervos en los bordes del bosque y en los pequeños claros del interior, mientras que al medio-

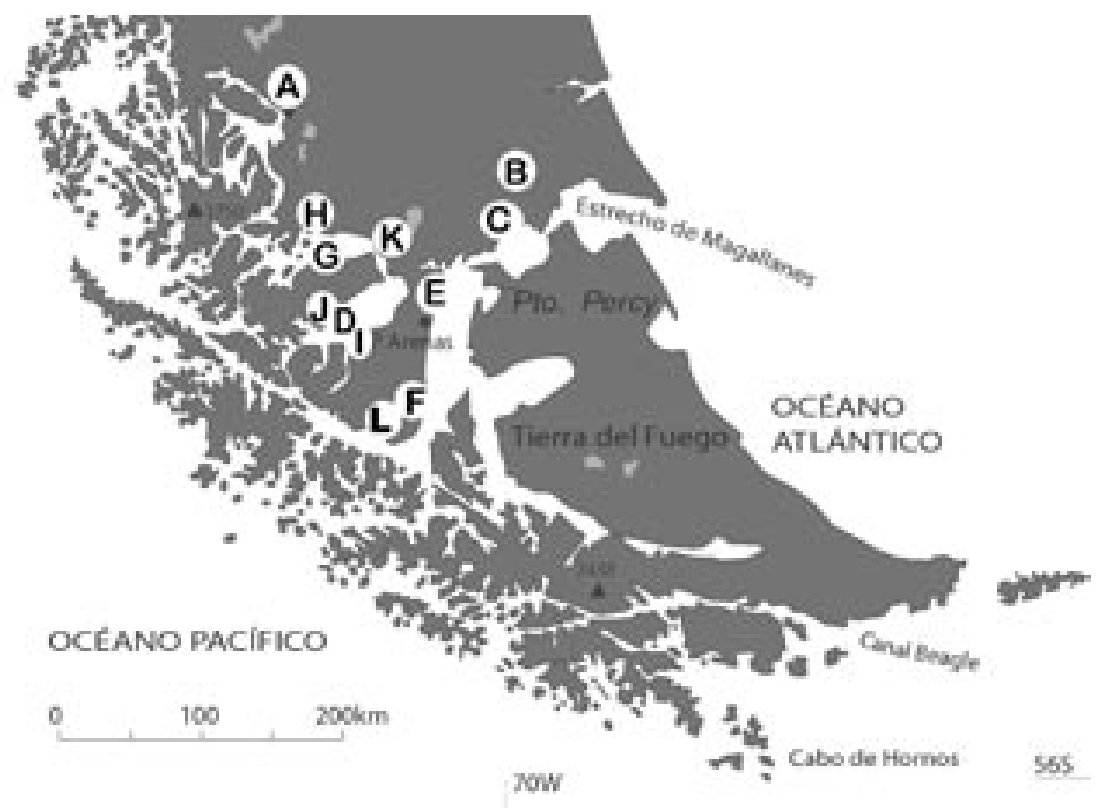

Fig. 1. Sitios arqueológicos del extremo austral de la Patagonia con presencia de restos de Hippocamelus bisulcus

A) Cueva Dos Herraduras Este, Cueva del Milodón, Cueva del Medio (Cervidae), y Cueva del Lago Sofía 4;

B) Cueva Fell; C) San Gregorio 10; D) Isla Englefield; E) Bahía Laredo-Cabo Negro; F) Bahía Buena, Punta Santa Ana; G) Punta Adelaida: sitio 24, Punta Garay: sitio 49; H) Isla Escapada: sitios 3 y 6, Arch. Señoret: 14 y 15, B. Rebolledo: sitios 25, 26, 28, 32, 33, B. Cable: sitios 34, 35 y 42; I) Puesto La Sal, Río Caleta 2; J) Martín Pescador 2, Cta. Eros 2, Cta. Alonso 2, Punta Eugenia 2; K) Río Verde 1; L) Sitio M3. 
día se los encontraba con frecuencia en la espesura de los bosques. El grado de confianza y temeridad demostrado por esos animales tradicionalmente tímidos era realmente de lo más extraordinario. Era muy evidente que desconocían totalmente al hombre. En una oportunidad, mientras estaba recorriendo los bosques con el arma en busca de alguna presa pequeña, me encontré con un macho adulto completamente desarrollado que estaba tranquilamente echado al pie de un árbol grande. Al detenerme para observarlo, se quedó bastante quieto por un momento y me miró, sin demostrar miedo en su expresión. Luego, poniéndose de pie lentamente se acercó caminando directamente hacia mí ese paso firme y medido característico de la familia. Todo en la actitud y porte del animal se parecía a una vaca o un caballo favorito cuando, tomando sol holgazanamente en el corral, se levanta y avanza lentamente para lamer la mano extendida del amo. Dejé que continuara esta exhibición de confianza hasta que se acercó a unos tres o cuatro metros de mí, cuando demostré mi indignidad al cambiar una carga pequeña por un tiro sólido que, luego de retroceder unos pasos, descargué con tal efecto que el hermoso animal cayó muerto casi a mis pies, víctima de una confianza equivocada depositada en un lugar no indicado (Hatcher, 2003:150-151).

Extraña confianza dado que el área en que habitaba el huemul no estaba exenta de predadores, el puma (Felis concolor) o el zorro colorado (Pseudalopex culpaeus) coexistían con ellos en la precordillera. Ambos predan sobre este animal aunque no lo hiciera sobre los adultos el zorro y sí el puma. Sin embargo, esta actitud de confianza era diferente en su rango geográfico más septentrional donde se lo cazaba (Wolffsohn 1910). Así lo evidencia la información arqueológica y etnográfica con un mayor aprovechamiento de pieles de huemul para las armaduras de combate entre los araucanos (Orbigny 1945), las botas de cuero de los pehuenches (Claraz 1864), toldos y tamangos (Perea 1989) y punzones sobre asta en el Neuquén (Silveira 1996).

La región de Magallanes ofrece un interesante ejemplo de la capacidad de modificación infligida por el hombre en el comportamiento del huemul. Cuando revisamos los relatos de los primeros navegantes observamos esta diferencia: tímido y confiado donde no se lo molestaba, o arisco y huidizo donde era cazado. En el siglo XVI, por ejemplo, Sarmien- to de Gamboa (1950) y Ladrillero (Pastells 1920) destacaban la facilidad de la caza del huemul en Última Esperanza y en Ciudad del Rey Don Felipe. Por el contrario, cuando los ingleses de la Beagle llegaron tres siglos más tarde a la misma región, los ciervos huían velozmente ante el primer disparo de sus armas (Fitz Roy 1839). Sin embargo, en algunas áreas boscosas y más resguardadas del hombre, el huemul conservaba su actitud de mansedumbre y así lo destacó Florence Dixie (1880) cuando en 1879 lo cazó en Torres del Paine. Más aún, a principios del siglo siguiente, algunos pobladores de Última Esperanza los acostumbraban a acercarse a las casas para darles de comer, sólo con no darle caza (Wolffsohn 1910). Esta diferencia de comportamiento también pudo verificarse más recientemente en el Parque Nacional "Bernardo O'Higgins", Chile. En 1991, Frid (2001) podía acercarse hasta cinco metros o menos a los huemules que estudiaba en el Estero Bernardo, en tanto que en 1995 no lo podía hacer a menos de 200 metros, según su opinión, atribuible a una mayor presión de caza.

Por otra parte, Julieta Gómez Otero (1986-87) ya había avanzado la hipótesis de que la presencia de restos de huemul en sitios arqueológicos de la estepa podría indicar una incursión cazadora hacia occidente. ¿Qué tan sostenida era dicha incursión en el tiempo?, era y es una cuestión difícil de evaluar aún. Por lo menos, durante el último cuarto del siglo XIX y en adelante, ciertos sectores parecen no haber sido frecuentados por las bandas de cazadores recolectores, ya no pedestres, sino ecuestres.

Los cazadores terrestres patagónicos australes eran eminentemente cazadores de guanaco, sobre todo a partir de la extinción de la megafauna hacia el límite Pleistoceno-Holoceno. Tanto así, que José Emperaire llega a hablar de una edad del guanaco en Patagonia (Emperaire, 1988). Por otra parte, los nómades marinos del archipiélago patagónico eran eminentemente cazadores de pinnípedos, clase utilizada desde las primeras ocupaciones a partir del Holoceno medio hasta la virtual extinción de estas poblaciones humanas en su modo nómada en el siglo XX. Sin embargo, en ciertos sectores como los distritos Otway, Skyring y Última Esperanza parece haber habido un aprovechamiento un poco más intensivo del huemul sin llegar nunca a ocupar el lugar de los pinnípedos (Legoupil, 1989 y 2000; San Román et al., 2002). 
No nos detendremos aquí en el registro de la presencia de restos de huemul en sitios arqueológicos precordilleranos y esteparios, tratada ya por Gómez Otero (1986-87) y Belardi y Gómez Otero (1998). $\mathrm{Ni}$ en la discusión acerca de los avances y retrocesos holocénicos del bosque, tratando de ligar el huemul con éste en una forma de silvodependencia.

Cabe destacar sí, los registros más occidentales de sitios arqueológicos del ámbito cazador terrestre. Para la zona austral, se trata del sitio taller junto a la laguna de los Choros (poco más al oeste de los $73^{\circ}$ de longitud), cerca del glaciar Tyndall en el Parque Nacional "Torres del Paine". Se trata de un sitio taller lítico bastante notable, por hallarse en un sector hoy en día muy húmedo, contándose con los primeros registros de ciprés de las Guaitecas (Pilgerodendrum uvifera) en sus cercanías. Debería tratarse de un sitio tardío aunque no histórico, dado que los fenómenos glaciales han estado activos allí bastante tardíamente.

De modo que por lo menos durante mediados del Holoceno un sector supuestamente apto para ser habitado por huemules, según lo muestran sus preferencias actuales, se habría encontrado esporádicamente habitado por el hombre. Poco más al este se encontraron boleadoras aisladas igualmente y puntas pequeñas apedunculadas en la orilla sur del río Serrano. No se han hallado sitios habitacionales en este sector como para ver qué papel jugó el huemul en la economía doméstica ligada a estas incursiones.

En la pampa, tal como lo señalara Gómez Otero (op. cit. 1986-87), hay esporádicos y escasos registros de restos de huemul a partir de los períodos III y IV de Bird (1993) esto es hacia el Holoceno Medio. No sabemos aún si esta provisión de tiempos históricos entre cazadores terrestres se debía a incursiones en su territorio o al aprovechamiento de huemules que se internaban en la estepa según lo ha establecido Díaz (1993, 2000).

En cuanto al papel de éste en la economía de los canoeros parece haber jugado un rol más importante, aunque nunca de la importancia alcanzada por los pinnípedos. El registro arqueológico de los alrededores de los mares de Otway y Skyring y en Última Esperanza muestra la presencia constante de huemul al lado de una mayoría de pinnípedos (Legoupil, op. cit., San Román et al. 2002). Cabe mencionar aquí el registro hecho por Robert FitzRoy en caleta Donkin, mar de Otway, de indios con capas del ciervo andino a los que bautizó "huemules" precisamente por ello (Fitz Roy 1933: 288-289).

También, en los archipiélagos del oeste, restos de huemul fueron encontrados en algunos sitios tardíos, tanto en Puerto Edén (Curry 1991) como al sur del golfo de Penas en el sitio Stuven 1 (Legoupil et al., en prensa Sextas Jornadas de Arqueología de la Patagonia), y en isla Madre de Dios. Así, algunas de las islas de la zona parecen haber sostenido pequeñas poblaciones de huemules, por lo menos en la isla Wellington donde la presencia de este animal fue confirmada por Emperaire (1963); pero en otras, algunos de sus restos pudieron ser llevados desde otros lugares lejanos en donde sí se hallaba presente.

\section{CONCLUSIONES}

La domesticación de cérvidos tiene una larga historia en Europa y Asia, y particularmente en regiones cercanas al Círculo Polar Ártico donde los cazadores primitivos probablemente comenzaron siguiendo a los rebaños de renos salvajes hasta conseguir progresivamente su amansamiento. A pesar de la mansedumbre y confianza del huemul hacia el hombre, éste no fue domesticado. Si bien Francisco Núñez de Piñeda y Bascuñán (en Monleon 1976) señaló que los araucanos criaban animales, especialmente auquénidos y huemules, aunque su número a la llegada de los españoles era escaso, esta práctica no fue común para la especie.

Ahora bien, para este proceso de domesticación el animal tiene que demostrar al cazador su utilidad, aunque no necesariamente llegue a depender de él para su supervivencia. El huemul no fue la presa principal de los grupos de cazadores recolectores de Patagonia, ni de los canoeros quienes mostraron un mayor interés por este recurso. Esto nos lleva al trabajo de Belardi y Gómez Otero (1998) sobre la anatomía económica de esta especie. De acuerdo al análisis de algunas partes esqueletarias de un huemul macho, su carne es magra y este factor, conjuntamente con la baja predictabilidad y su baja densidad poblacional, pudieron ser determinantes para la baja explotación.

Podríamos hipotetizar que los canoeros practicaron la caza del huemul en forma puntual en tiempo y espacio para optimizar la relación costo-beneficio. En tiempo porque se vincularía especialmente con el invierno cuando los huemules bajan de tierras más altas o cuando beben agua en 
lagos y ríos, y en espacio porque se aprovecharían principalmente los sitios de procesamiento ubicados en las desembocaduras de los ríos para su captura. Esta técnica de caza cerca del agua fue observada por Bird (en Gusinde 1991) y Emperaire (1963).

Históricamente el huemul ha mostrado un comportamiento de peculiar mansedumbre hacia el hombre respecto a otros ciervos. Su conformación anatómica, extremidades relativamente cortas, músculos cortos y poderosos, extremidades posteriores en semiflexión, su incapacidad de correr grandes distancias pero su velocidad en distancias cortas, sugieren su adaptación a terrenos accidentados y fuertes pendientes (Aldridge com. pers., 1999). Además, la coloración del pelaje y de las crías también lo hacen más asociado a la vegetación de áreas mésicas (Aldridge com. pers., 1999). Entonces, es muy probable que inicialmente este ciervo haya tenido una distribución más amplia en Magallanes, y que una mayor densidad humana y presión de caza lo haya empujado a buscar refugio en el bosque virgen magallánico y cada vez a más altura en las montañas. El etnolímite que mencionamos anteriormente correspondería entonces a la frontera más allá de la cual el huemul encontró su refugio y supervivencia.

Si bien la densidad poblacional del huemul no fue alta, en relación con la del guanaco, y su comportamiento hacia el hombre en la primera etapa de contacto fue de extrema confianza y vulnerabilidad, la especie no se extinguió. Para ello pudieron intervenir varios factores: i) el huemul nunca predominó en la dieta de los cazadores sino que fue un recurso alimenticio complementario, ii) las especies animales alternativas eran abundantes, iii) el paisaje escarpado $y$ boscoso le ofrecieron una baja visibilidad y accesibilidad, y iv) la baja densidad poblacional humana en algunas regiones, como Magallanes, configuró una importante reserva de la especie.

\section{BIBLIOGRAFÍA}

BELARDI, J. B. y J. GÓMEZ OTERO. 1998. Anatomía económica del huemul (Hippocamelus bisulcus): una contribución a la interpretación de las evidencias arqueológicas de su aprovechamiento en Patagonia. Anales del Instituto de la Patagonia, Serie Ciencias Humanas 26:195-207.

BIRD, J. 1993. Viajes y Arqueología en Chile Austral. Editado por John Hysloop. Ediciones de la Universidad de Magallanes. 283 pág. Punta Arenas, Chile.
CLARAZ, M. G. 1864. Sur l'Equus bisulcus de Molina. Lettre de M. G. Claraz à M. H. De Saussure. Extrait de la Revue et Magasin de Zoologie.

CURRY, P. 1991. Distribución de sitios e implicaciones para la movilidad de los canoeros en el canal Messier. Anales del Instituto de la Patagonia, Serie Ciencias Humanas 20:145-154.

DÍAZ, N.I. 1993. Changes in the range distribution of Hippocamelus bisulcus in Patagonia. Zeitschrift für Säugetierkunde 58:344-351.

- 2000. El huemul (Hippocamelus bisulcus Molina, 1782): Una perspectiva histórica. En: El huemul patagónico: un misterioso cérvido al borde de la extinción. L.O.L.A. (ed.), Monografía 3, Buenos Aires.

DIXIE, F. C. 1880. Across Patagonia. R. Bentley \& Son, London.

EMPERAIRE, J. 1963. Los nómades del mar. Ediciones de la Universidad de Chile, Santiago.

- 1988. Paisajes y hombres prehistóricos de la Patagonia. Anales del Instituto de la Patagonia, Serie Ciencias Humanas 18:79-94.

FITZ ROY, R. 1839 (1933). Narrative of the Surveying of H.M.S. Adventure and Beagle between the years 1826 and 1836. Henry Colburn, London.

FRID, A. 2001. Habitat use by endangered huemul (Hippocamelus bisulcus): cattle, snow, and the problem of multiple causes. Biological Conservation 100:261-267.

GÓMEZ OTERO, J. 1986-1987. Investigaciones arqueológicas en el alero Potrok-Aike (Provincia de Santa Cruz): Una revisión sobre los períodos IV y V de Bird. Relaciones de la Sociedad Argentina de Antropología 17(1):17319, Buenos Aires.

GUSINDE, M. 1991 (1974). Los indios de Tierra del Fuego. Vol. II, Los Halakwulup. Centro Argentino de Etnología Americana, Buenos Aires.

HATCHER, J. 2003 (1903). Cazadores de huesos en la Patagonia, University Press, John Bell Hatcher. Zagier \& Urruty Ed., Ushuaia, Argentina.

LEGOUPIL, D. 1989. Ethno-archéologie dans les Archipels de Patagonie: les nomades marins de Punta Baja. Recherches sur les Civilisations (ed.), París.

- 2000. El sistema socioeconómico de las nómadas del mar de Skyring. Anales del Instituto de la Patagonia, Serie Ciencias Humanas 28:81-120.

LEGOUPIL, D., P. BEAREZ, S. LEPETZ, M. SAN ROMÁN y K. SALAS. 2007. De la pesca a la caza: evolución de un campamento en medio de los archipiélagos de Patagonia. Actas de las VI Jornadas de Arqueología de la Patagonia. Punta Arenas, Chile. Octubre 2005. En prensa. 
MARSHALL, L.G. 1984. Who killed cock robin? In: Quaternary extinctions: A prehistoric revolution, P.S. Martin and R.G. Klein (Eds.), University of Arizona Press, Tucsan, AZ, pp. 791-792.

MONLEON, P., J. 1976. Los araucanos: etnohistoria y arqueología. Tesis para obtener la licenciatura en Prehistoria. Universidad de Chile, Santiago.

ORBIGNY, A. D. D'. 1945. Viaje a la América Meridional, Brasil, República del Uruguay, República Argentina, la Patagonia, República de Chile, República de Bolivia, República del Perú, realizado de 1826 a 1833. Futuro, Buenos Aires.

PASTELLS, P. 1920. El descubrimiento del Estrecho de Magallanes. Sucesores de Rivadeneyra S.A. Artes Gráficas. Madrid.
PEREA, E. 1989. Y Félix Manquel dijo. Textos Ameghinianos. Fundación Ameghino.

SAN ROMÁN, M; F. MORELLO y A. PRIETO. 2002. Nuevos antecedentes sobre la explotación de recursos faunísticos en el mar de Otway y canales adyacentes. Anales del Instituto de la Patagonia, Serie Ciencias Humanas 30:147-154.

SARMIENTO DE GAMBOA, P. 1950. Viajes al Estrecho de Magallanes. Emecé, Buenos Aires, 2 vol.

SILVEIRA, M.J. 1996. Alero Los Cipreses (Provincia del Neuquén, República Argentina). En: Arqueología. Sólo Patagonia. J. Gómez Otero, (ed.), CENPAT-CONICET, Puerto Madryn.

WOLFFSOHN, J.W. 1910. Notas sobre el huemul. Revista Chilena de Historia Natural 14:227-234.

Referencias

Registros de la actitud del huemul hacia el hombre

\section{Tímido y confiado}

A. Junín de los Andes

B. Río Richards

C. Río Los Antiguos

D. Lago Belgrano

E. Glaciar Jorge Montt

F. Lago San Martín

G. Estero Bernardo (en 1991)

H. Río Chico

I. Lago Argentino

J. Torres del Paine

K. Última Esperanza

L. Punta Santa Ana (en el siglo XVI)

\section{Arisco}

M. Río Caleufú

N. 16 de Octubre

O. Río Frías

P. Río Baker

Q. Bahía Fortescue

(G) Estero Bernardo (en 1995)

(L) Punta Santa Ana (en el siglo XIX)

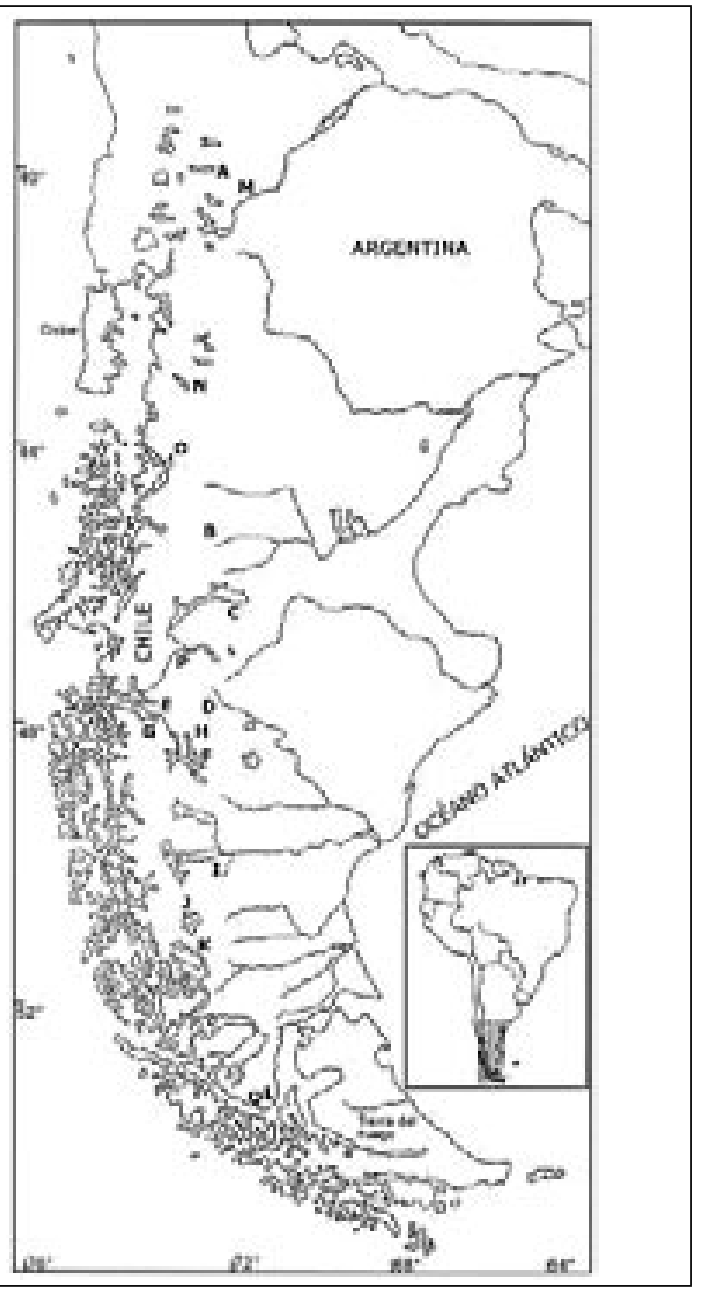

